\title{
A Rare Case of Solitary Parathyroid Adenoma presenting with Recurrent Pancreatitis detected by Dual-phase Single-isotope Imaging (Technetium Sestamibi and Technetium Thyroid Scan) with SPECT-CT
}

\author{
${ }^{1}$ Arun R John, ${ }^{2}$ Anurag Jain, ${ }^{3}$ Madan G Vishnoi, ${ }^{4}$ Dharmesh Paliwal, ${ }^{5}$ Amit Sharma, ${ }^{6}$ Neeraj Kumar, ${ }^{7}$ Indra P Dubey
}

\begin{abstract}
Primary hyperparathyroidism is a rare cause of acute pancreatitis with a rather uncommon association according to existing literature. We present a rare case of recurrent pancreatitis with primary hyperparathyroidism resulting from a solitary parathyroid adenoma detected by a dual-phase singleisotope imaging protocol using technetium pertechnetate and technetium sestamibi followed by a single-photon emission computed tomography (SPECT)-CT. The case demonstrates the requirement of a high index of suspicion of primary hyperparathyroidism as one of the etiologies in patients presenting with recurrent pancreatitis. This case highlights the role of dualphase single-isotope imaging (technetium pertechnetate and technetium sestamibi scan) along with SPECT-CT for precise anatomical localization of a parathyroid adenoma, which is of paramount importance to the surgeon for meticulous planning and execution of the surgical procedure.
\end{abstract}

Keywords: Dual-phase single-isotope imaging protocol, Primary hyperparathyroidism, Recurrent pancreatitis, Singlephoton emission computed tomography, Solitary parathyroid adenoma, Technetium sestamibi.

How to cite this article: John AR, Jain A, Vishnoi MG, Paliwal D, Sharma A, Kumar N, Dubey IP. A Rare Case of Solitary Parathyroid Adenoma presenting with Recurrent Pancreatitis detected by Dual-phase Single-isotope Imaging (Technetium Sestamibi and Technetium Thyroid Scan) with SPECT-CT. World J Endoc Surg 2016;8(2):175-178.

Source of support: Nil

Conflict of interest: None

\section{INTRODUCTION}

Primary hyperparathyroidism is a rare cause of recurrent pancreatitis, especially in young adult males. Primary hyperparathyroidism, usually presenting as a solitary adenoma, is more prevalent in females above the age of

\footnotetext{
${ }^{1,3,4,6}$ Resident, ${ }^{2,5,7}$ Faculty

${ }^{1-7}$ Department of Nuclear Medicine, Army Hospital Research and Referral, Dhaula Kuan, New Delhi, India

Corresponding Author: Anurag Jain, Faculty, Department of Nuclear Medicine, Army Hospital Research and Referral, Dhaula Kuan, New Delhi, India, Phone: +9540158095, e-mail: triplea. jain@gmail.com
}

50 years, with a known prevalence of $21 / 1000 .{ }^{1}$ The incidence is less than $5 \%$ in adolescents and young adults aged 12 to 28 years, ${ }^{2}$ A solitary adenoma is responsible for $80 \%$ of cases of primary hyperparathyroidism. ${ }^{3}$ We present a case of recurrent pancreatitis associated with primary hyperparathyroidism. Dual-phase single-isotope planar imaging with technetium pertechnetate and technetium sestamibi was performed on two separate days along with single-photon emission computed tomography (SPECT)-CT to detect the precise anatomical localization of a solitary parathyroid adenoma.

\section{CASE REPORT}

A 25-year-old male presented with a history of abdominal and recurrent episodes of vomiting for a duration of 12 hours. The pain was acute in onset, located in the epigastrium initially and later spread to the entire upper abdomen, radiating to the back and partially relieved on bending forward. The pain was dull aching in character, which gradually increased in intensity over a period of 2 hours and thereafter remained unaltered in intensity. The pain was also associated with multiple episodes of nonprojectile, nonbilious, and nonblood-stained vomiting. The symptoms were also associated with abdominal distension and loss of appetite. There was no history of fever, jaundice, cough, dysuria, hematuria, upper gastrointestinal bleed, or drug intake. The patient gave a history of two similar episodes in the preceding 2 years. Clinical examination revealed tenderness and guarding in the epigastrium along with tachycardia (pulse 136/min). Initial investigations revealed anemia (hemoglobin $11.2 \mathrm{~g} / \mathrm{dL})$, elevated serum amylase (1100 U/L), elevated serum lipase (2860 U/L), and hypercalcemia $(12.2 \mathrm{mg} / \mathrm{dL}$, reference range: 8.9 to $10.3 \mathrm{mg} / \mathrm{dL}$ ). The liver function tests, serum proteins, albumin, lipid profile, electrolytes, and phosphorus levels were within normal limits. Ultrasonography of abdomen revealed a diffusely enlarged pancreas along with distended gallbladder. There was no evidence of necrosis or calcification. Contrast-enhanced computed tomography of abdomen performed subsequently revealed a bulky pancreas with homogenous 
enhancement and peripancreatic fat stranding. The main pancreatic duct was not dilated and no stones were found. Based on the above findings, a provisional diagnosis of acute pancreatitis was made. The etiology was suspected to be likely due to hypercalcemia. The patient was managed conservatively for the same. Further detailed investigations were carried out to confirm and manage the likely etiology. In view of hypercalcemia, the patient was investigated to determine the serum parathormone level, which was found to be elevated (147.55 pg/mL, reference range: 10 to $65 \mathrm{pg} / \mathrm{mL}$ ) with normal thyroid function tests. An ultrasound of the neck revealed a colloid cyst in the right lobe of thyroid. The patient was sent to the Department of Nuclear Medicine and was subjected to a dual-phase single-isotope planar imaging study with technetium pertechnetate and technetium sestamibi on two separate days (Fig. 1). The technetium thyroid scan on day 1 revealed a large photopenic area in the inferior pole of the right lobe of thyroid. The technetium sestamibi study performed on a subsequent day revealed a focus of increased tracer uptake in the inferior pole of the right lobe of the thyroid, with a complete tracer washout from the rest of the thyroid after 120 minutes of study. The planar imaging was positive for parathyroid adenoma at the inferior pole of the right lobe of thyroid. This finding was corroborated with SPECT-CT, which further delineated and localized the lesion to the inferior pole of the right lobe of thyroid (Fig. 2). Subsequent magnetic resonance imaging also corroborated the above findings.

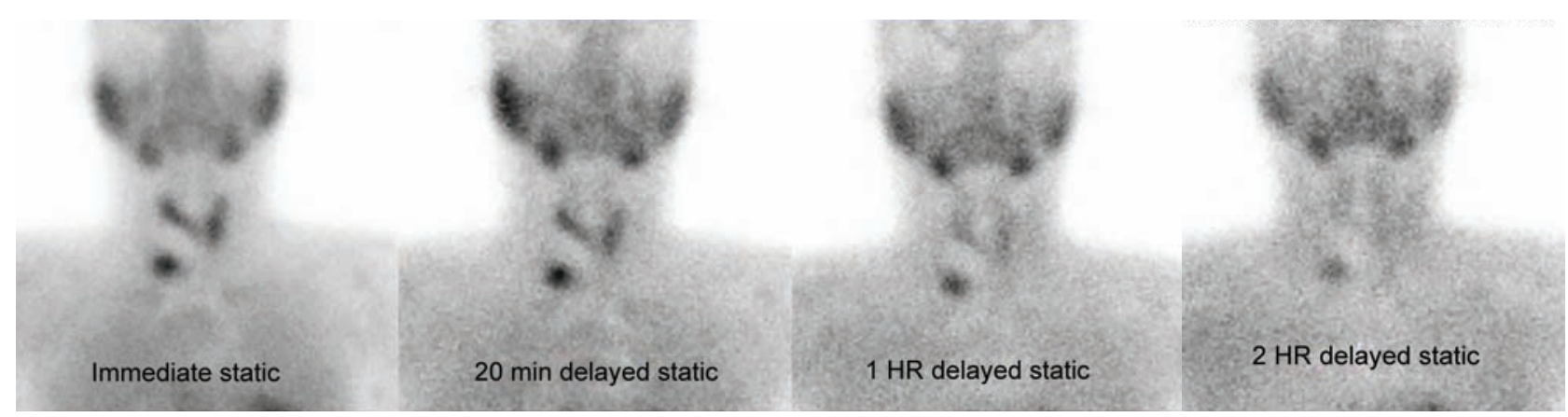

Fig. 1: Sequential planar static images post-technetium 99m sestamibi injection, showing a large cold nodule in the right lobe of thyroid and normal thyroid tissue. In the sequential images, there is washout of tracer from the normal thyroid with a focus with retained tracer uptake in the inferior pole of the thyroid
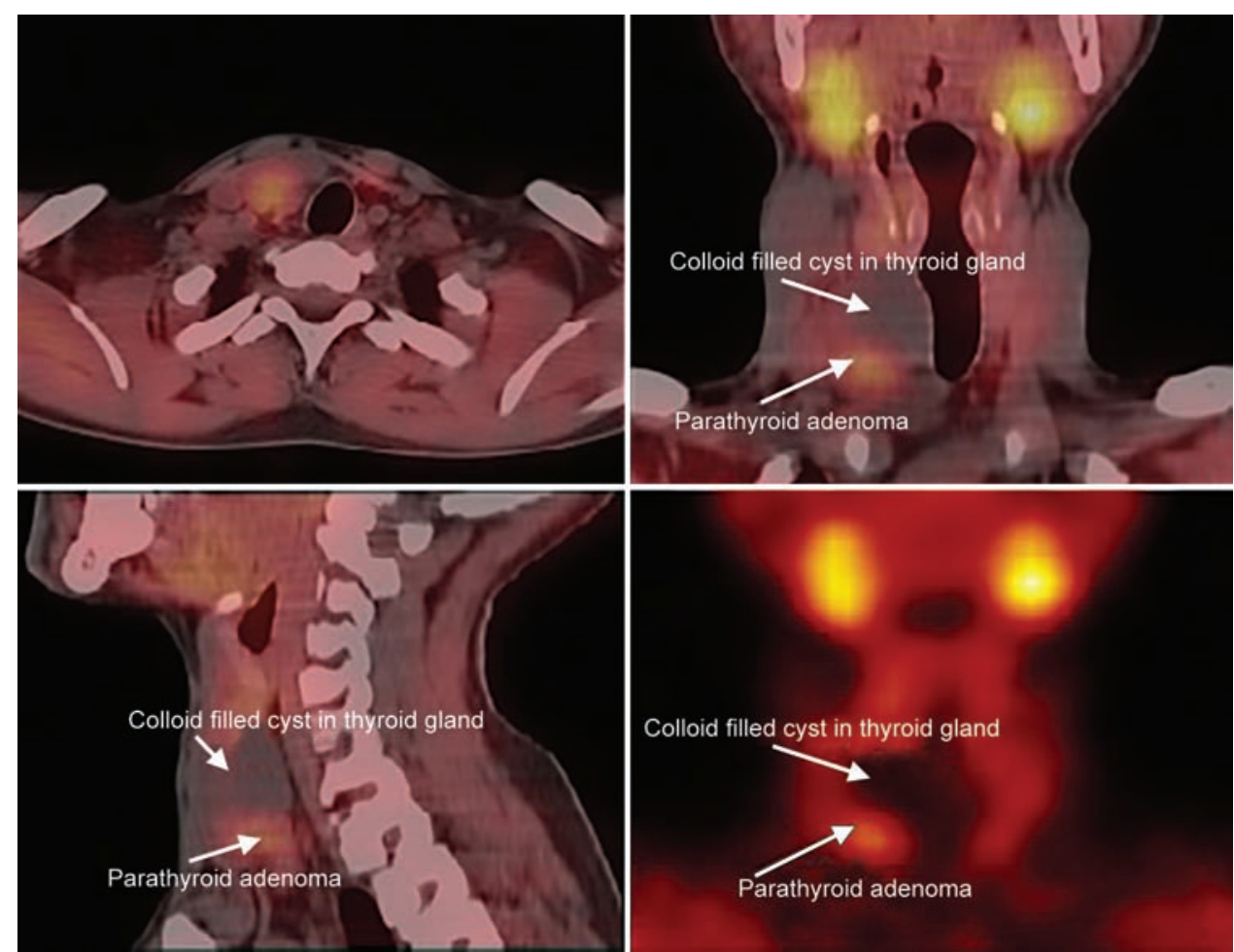

Fig. 2: The SPECT-CT images in axial, coronal, sagittal sections and pure SPECT showing a well-defined lesion with increased tracer uptake at the inferior pole of thyroid gland 


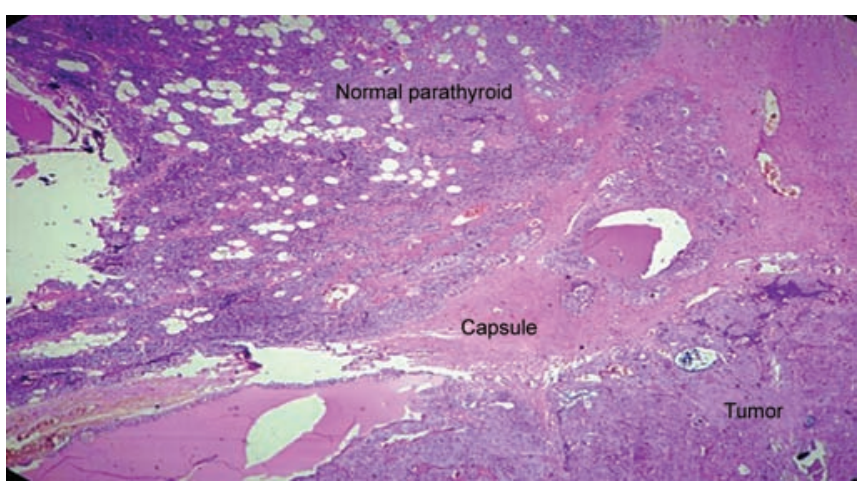

Fig. 3: Hematoxylin and eosin-stained slide from the surgical specimen showing an encapsulated parathyroid tumor with scant adipose tissue

The patient was, therefore, taken up for surgical removal of the parathyroid adenoma. Postoperative histopathological examination confirmed the presence of parathyroid adenoma (Figs 3 and 4). Following the surgery, the patient was asymptomatic and serum amylase, lipase, and calcium levels returned to normal limits. A postoperative parathormone assay was also normal $(0.01 \mathrm{pg} / \mathrm{mL})$.

\section{DISCUSSION}

We have presented a rare case of recurrent pancreatitis due to primary hyperparathyroidism resulting from a solitary parathyroid adenoma detected by a dual-phase single-isotope imaging protocol using technetium pertechnetate and technetium sestamibi. The diagnosis of primary hyperparathyroidism depends on the laboratory confirmation of increased serum calcium and parathormone levels. The incidence of acute pancreatitis associated with hyperparathyroidism is reported to be $<10 \%{ }^{4,5}$ According to existing literature, the association between pancreatitis and primary hyperparathyroidism appears to be rather uncommon. The likely mechanism of hyperparathyroidism leading to acute pancreatitis is that hypercalcemia may cause activation of trypsinogen to trypsin, resulting in autodigestion of the pancreas. Subsequently, it may also lead to the formation of pancreatic calculi, pancreatic ductal obstruction, and subsequent attacks of acute or chronic pancreatitis. ${ }^{6}$ Studies have also shown that hypercalcemia in combination with genetic variants in serine protease inhibitor kazal type 1 (SPINK1) and cystic fibrosis transmembrane conductance regulator (CFTR) genes can increase the risk of developing acute pancreatitis in patients with primary hyperparathyroidism. ${ }^{7}$ A study by Bai et $\mathrm{al}^{8}$ reviewed the association between acute pancreatitis and primary hyperparathyroidism. The study reported that the published cohorts of patients were subject to bias, because serum calcium

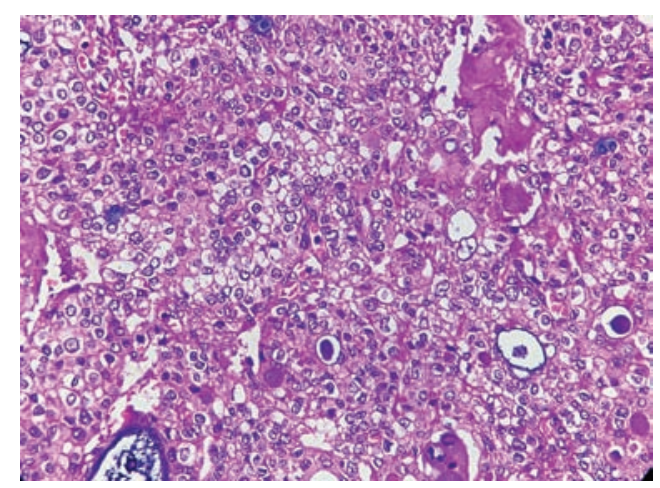

Fig. 4: Hematoxylin and eosin-stained slide from the surgical specimen in high magnification (100×) showing parathyroid adenoma cells with minimal nuclear pleomorphism and no mitotic figures

screening was not universally performed among all control patients. They also concluded that hypercalcemia may contribute to pancreatitis in these cases, along with genetic or environmental insults. The Mayo Clinic experience between 1950 and 1975 found that out of 1,153 patients with primary hyperparathyroidism, only $17(1.5 \%)$ had coexisting pancreatitis. Other factors of possible etiologic significance in pancreatitis, such as gallstones or alcohol abuse, were present in 11 of 17 patients. ${ }^{9}$ Misgar et $\mathrm{a}^{10}$ reported the case of a 32-year-old man who was diagnosed to have primary hyperparathyroidism during the fourth attack of pancreatitis and his postoperative recovery period was uneventful. Agarwal et al ${ }^{11}$ reported that pancreatitis was found to be associated with primary hyperparathyroidism in 6 of 87 patients (6.8\%), and despite its rarity, a cause and effect relationship was still suggested by the fact that parathyroidectomy seems to prevent recurrence of pancreatitis. Krishnamurthy et $\mathrm{al}^{12}$ also reported the association between giant parathyroid adenoma and recurrent pancreatitis. They effectively pointed out that preoperative localization techniques that would include a parathyroid scintigraphy and a focused surgical intervention are important to resolve complications and improve clinical outcomes.

Precise preoperative localization of the parathyroid adenoma is essential for surgical management. The use of single-isotope dual-phase scintigraphic imaging technique is based on the observation that technetium sestamibi washes out more rapidly from the thyroid gland than from the hyperfunctioning parathyroid glands. ${ }^{13}$ This is due to the increased number of mitochondria in the parathyroid adenomatous cells that concentrates the technetium sestamibi. The use of SPECT-CT along with technetium sestamibi is more superior than the planar study alone, in the accurate preoperative localization of the hyperfunctioning pathologic glands, especially in patients suffering from multiglandular disease with primary hyperparathyroidism. ${ }^{14,15}$ 


\section{CONCLUSION}

This case further reiterates the rare association between primary hyperparathyroidism and recurrent pancreatitis with the requirement of a high index of suspicion to detect the same along with the role of early serum calcium estimation in cases of acute pancreatitis. The case also highlights the role of dual-phase single-isotope imaging (technetium sestamibi and technetium thyroid scan) in clinching the etiological diagnosis. The SPECT-CT component in the study has an added benefit of precise anatomical localization of the tumor in the parathyroid glands, which is of paramount importance to the surgeon for planning and execution of the surgery and also to improve the surgical outcome.

\section{ACKNOWLEDGMENTS}

Authors would like to thank the Departments of Gastroenterology, Endocrinology, Radiodiagnosis and Pathology, Army Hospital Research and Referral, New Delhi for their immense help in preparing this case report.

\section{REFERENCES}

1. Adami S, Marcocci C, Gatti D. Epidemiology of primary hyperparathyroidism in Europe. J Bone Miner Res 2002 Nov;17(2):N18-N23.

2. Yeh MW, Ituarte PH, Zou HC, Nishimoto S, Liu I-L, Harari A, Adams AL. Incidence and prevalence of primary hyperparathyroidism in a racially mixed population. J Clin Endocrinol Metab 2013 Mar;98(3):1122-1129.

3. Udelsman R. Six hundred fifty-six consecutive explorations for primary hyperparathyroidism. Ann Surg 2002 May;235(5):665-670. Discussion 70-72.

4. Kunte AR, Dube VS, Balwantkar SS, Kulkarni KK. Parathyroid adenoma in a young female presenting as recurrent acute pancreatitis with a brown tumor of the mandible - A case study. Int J Surg Case Rep 2015;7C:10-15.
5. Tun-Abraham ME, Obregon-Guerrero G, Romero-Espinoza L, Valencia-Jimenez J. Acute pancreatitis associated with hypercalcaemia. Cir Cir 2015 May-Jun;83(3):227-231.

6. Jacob JJ, John M, Thomas N, Chacko A, Cherian R, Selvan B, Nair A, Seshadri M. Does hyperparathyroidism cause pancreatitis. A south Indian experience and a review of published work? ANZ J Surg 2006 Aug;76(8):740-744.

7. Felderbauer P, Karakas E, Fendrich V, Bulut K, Horn T, Lebert R, Holland-Letz T, Schmitz F, Bartsch D, Schmidt WE. Pancreatitis risk in primary hyperparathyroidism: Relation to mutations in the SPINK1 trypsin inhibitor (N34S) and the cystic fibrosis gene. Am J Gastroenterol 2008 Feb;103(2):368-374.

8. Bai HX, Giefer M, Patel M, Orabi AI, Husain SZ. The association of primary hyperparathyroidism with pancreatitis. J Clin Gastroenterol 2012 Sep;46(8):656-661.

9. Bess MA, Edis AJ, van Heerden JA. Hyperparathyroidism and pancreatitis. Chance or a causal association? JAMA 1980 Jan;243(3):246-247.

10. Misgar RA, Mathew V, Pandit K, Chowdhury S. Primary hyperparathyroidism presenting as recurrent acute pancreatitis: a case report and review of literature. Indian J Endocrinol Metab 2011 Jan-Mar;15(1):54-56.

11. Agarwal A, George RK, Gupta SK, Mishra SK. Pancreatitis in patients with primary hyperparathyroidism. Indian J Gastroenterol 2003 Nov-Dec;22(6):224-225.

12. Krishnamurthy A, Raghunandan GC, Ramshankar V. A rare case of giant parathyroid adenoma presenting with recurrent episodes of pancreatitis. Indian J Nucl Med 2016 Jan-Mar;31(1):36-38.

13. Taillefer R, Boucher Y, Potvin C, Lambert R. Detection and localization of parathyroid adenomas in patients with hyperparathyroidism using a single radionuclide imaging procedure with technetium-99m-sestamibi (double-phase study). J Nucl Med 1992 Oct;33(10):1801-1807.

14. Kim YI, Jung YH, Hwang KT, Lee HY. Efficacy of ${ }^{99} \mathrm{mTc}-$ sestamibi SPECT/CT for minimally invasive parathyroidectomy: Comparative study with ${ }^{99} \mathrm{mTc}$-sestamibi scintigraphy, SPECT, US and CT. Ann Nucl Med 2012 Dec;26(10):804-810.

15. Chakraborty D, Mittal BR, Harisankar CN, Bhattacharya A, Bhadada S. Spectrum of single photon emission computed tomography/computed tomography findings in patients with parathyroid adenomas. Indian J Nucl Med 2011 JanMar;26(1):52-55. 